\title{
Experimento de medição do nível de Atenção do Estudante: o uso da Mídia Interativa como Estímulo Resposta
}

\author{
Andreia Solange Bos ${ }^{1}$, Michelle Câmara Pizzato ${ }^{1}$, Milton Antônio Zaro \\ ${ }^{1}$ Instituto Federal do Rio Grande do Sul (IFRS) \\ Porto Alegre - RS - Brazil \\ ${ }^{2}$ Universidade Federal do Rio Grande do Sul (UFRGS) \\ Porto Alegre RS - Brazil \\ \{andreia.bos\}@gmail.com, michelle.pizzato@poa.ifrs.edu.br, \\ zarodufrgs.br
}

\begin{abstract}
Resumo. Os tipos de atenção estudados pela literatura cientifica estão se tornando cada vez mais consistentes com as atividades do ser humano. Uma interação eficaz pode fazer com que a atenção seja usada como um recurso de estímulo resposta. Por ser um recurso importante, a atenção deve ser medida com indicadores quantitativos. Um método usado para medir isso é a aquisição do processamento e interpretação do tipo de onda EEG (Eletroencefalograma). O artigo apresenta um estudo comparativo entre métodos clássicos e métodos interativos para investigar a atenção e mensura os resultados observados.
\end{abstract}

Palavras Chave: ondas cerebrais, mídias interativas, atenção.

\section{Student Attention level measurement experiment: the use of interactive media as an answer stimulus}

\begin{abstract}
The types of attention studied in the scientific literature are becoming increasingly consistent with human activities. Effective interaction can cause attention to be used as a response stimulus resource. For an important resource, attention should be measured with quantitative indicators. One method used to measure this is the processing capture and interpretation of the EEG (electroencephalogram) wave type. The article presents a comparative study between classical and interactive methods to investigate attention and measure observed results.
\end{abstract}

Keywords: brain waves, interactive media, attention.

\section{Introdução}

Nos dias atuais a sociedade passa por constante transformação em que o uso das tecnologias de informação e comunicação tornou-se essencial em nosso estilo de vida. Dispositivos móveis em sala de aula não são mais um paradigma para professores e alunos. Por esta razão, vários educadores makers implementam várias aplicações educacionais. Entre esses sistemas, a maioria são gratuitos ou podem ser usados em um 
telefone celular. Assim a utilização não apresenta restrição nem para estudantes nem para os educadores. Nas atividades de aprendizagem, a característica mais importante é a atenção. Um processo ou ato de se concentrar em um ou mais fatores do ambiente em que os sentidos entram em contato.

Do ponto de vista da psicofisiologia a atenção tem dois componentes importantes: A atenção concentrada, usada em áreas dentro da esfera da interface cérebro computador, correspondendo a capacidade do cérebro de comandar e controlar a atividade motora. (Izquierdo, 2016). E a atenção cognitiva que se manifesta no campo da aprendizagem, que é a capacidade do cérebro para aprender. A atenção é diferenciada com base em atributos, dependendo da genética, da estrutura das atividades realizadas, experiência, motivação. As características da atenção são: Volume de atenção, estabilidade da atenção, mobilidade da atenção, distinção de atenção e atenção concentrada (Xiao, Zhang, 2015). A definição de atenção vai além de uma função simples e pode ser entendida como o direcionamento seletivo da consciência, que filtra e descarta informações indesejadas. As funções atencionais podem ser classificadas como atenção seletiva, ligada a preferência (seleção) de alguns estímulos em relação a outros. Atenção dividida que permite responder a duas tarefas de atenção seletiva e Atenção sustentada que é definida como a capacidade de manter a atenção de forma consistente durante determinado tempo e é objeto da presente investigação. Essa capacidade valoriza mais o primeiro momento conhecido como captura da concentração do estudante (atenção sustentada), a partir da qual os momentos subsequentes são revelados (Tokuhama- Espinosa, 2008).

O vídeo interativo é uma ferramenta instrucional versátil que ajuda os educadores a envolver os alunos na aprendizagem ativa (Bos et al., 2019c). Os vídeos interativos permitem que os educadores enviem seus próprios vídeos ou localizem vídeos públicos no YouTube e outros sites de compartilhamento. Elementos interativos, como avaliações, checklist e muito mais podem ser adicionados.

Para ajudar os alunos a tirar o máximo proveito de um vídeo educacional, é importante fornecer ferramentas para ajudá-los a processar as informações. Existem maneiras de fazer isso de forma eficaz, uma abordagem é o uso da plataforma H5P (Bos et al., 2019d).

Para esse estudo nos apoiamos na teoria cognitiva da aprendizagem multimídia, observando que a memória de trabalho possui dois canais para aquisição e processamento de informações: um canal visual e um canal de processamento auditivo/ verbal (Mayer, 2008).

Este artigo descreve como os alunos de Educação em Ciências usam os métodos clássicos (vídeo convencional) e métodos interativos (vídeos interativos). No estudo pretende-se investigar os tipos de atenção que os alunos desenvolvem com estímulos resposta. A ferramenta foi adaptada a várias formas de instrução para atender ao objetivo da investigação. O estudo foi determinar por meio de sinais de $\mathrm{EEG}^{1}$, quais dos métodos abordados estão associados a atenção sustentada.

\section{Revisão da Literatura}

\footnotetext{
${ }^{1}$ EEG: Eletroencefalografia é um método de monitoramento eletrofisiológico que é utilizado para registrar a atividade elétrica do cérebro.
} 
O streaming de vídeo é um ótimo ponto de partida para ensinar conceitos do ensino de ciências, mas observar passivamente um vídeo não é cognitivo e desafiador (Cherrett, 2009). A maioria dos vídeos não ensina efetivamente o material aos alunos. Os elementos interativos alteram a própria natureza dos vídeos on line, transformando-os em materiais de aprendizado passivo para ativo. Segundo Leeder 2000, essa interatividade é caracterizada por termos como navegar, investigar, explorar, escolher e fazer. A responsabilidade é transferida para o usuário.

Depois que os alunos enviam suas respostas, o vídeo exibe a resposta correta com explicação de texto. Quando perguntadas sobre suas impressões, a maioria dos estudantes afirmou que os vídeos interativos eram extremamente benéficos (Cherret, 2009). MacKenzie e Ballard 2015, cita que o uso de atividades interativas on line individua is pode melhorar os resultados dos exames.

Mackenzie 2015, em seu estudo descobriu que o aumento do envolvimento dos alunos com o material levou a melhores resultados nos exames e concluíram que esses tipos de suplementos são promissores para os alunos e tem um bom desempenho no curso. Pontuações de teste aprimoradas e um melhor foco também foram evidenciadas por (Szpunar, Khan e Schacter, 2013).

Lawson e colegas examinaram o impacto das perguntas orientadoras na aprendizagem dos alunos em um vídeo sobre psicologia social (Lawson, 2006). Com base no trabalho de Kreiner 1997, eles fizeram com que os alunos de um curso assistissem ao vídeo sem instruções especiais, enquanto os alunos de outro curso receberam oito perguntas norteadoras a serem consideradas enquanto assistiam. Os alunos que responderam as perguntas norteadoras obtiveram uma pontuação significativamente maior em um teste posterior.

Guo et al examinaram o tempo que os alunos assistiam a vídeos em streaming dentro de quatro Moocs, analisando os resultados. Eles observaram que o tempo médio de envolvimento para os vídeos com menos de seis minutos foi próximo de $100 \%$, ou seja os alunos tendem a assistir o vídeo inteiro. A medida que os vídeos aumentavam, o engajamento com vídeos de 9 a 12 minutos diminuía e passava a 50\% de tempo médio de engajamento. Já os vídeos com 12 a 40 minutos era de aproximadamente 20\%. O tempo médio com máximo envolvimento foi de seis minutos. Fazer vídeos com mais de 6 minutos é um esforço desperdiçado pelo educador (Guo, 2014).

Richard Mayer comenta o princípio da personalização, o uso da linguagem conversacional em vez da formal durante a elaboração da multimídia, demonstrando ter um grande efeito no aprendizado dos alunos, talvez porque um estilo de conversação incentive os alunos a desenvolver um senso de parceria, que conduz para um maior engajamento (Mayer, 2008).

Wei \& Ma (2016) utilizaram o NeuroSky para investigar as influências da atenção visual e do tempo de leitura em crianças e adultos enquanto liam álbuns de imagens impressas. O estudo investigou a relação entre atenção visual e tempo de leitura usando um dispositivo eletroencefalográfico móvel. O dispositivo Neurosky usa um sensor seco de canal único, que mede a atenção dos participantes no ambiente de leitura do mundo real.

Ferramentas como o H5p, podem permitir que os educadores incorporem perguntas diretamente no vídeo e dê um feedback com base na resposta do aluno. Bos et al (2019b), 
compararam os efeitos da atenção com leitura de texto e o uso de vídeos interativos, constatando que perguntas incorporadas no vídeo melhoraram o desempenho dos alunos nos testes de atenção.

A pesquisa em neurociência aborda o conhecimento sobre o cérebro e particularmente os sinais elétricos emitidos pelos neurônios. As frequências e padrões desses sinais elétricos podem ser medidos colocando-se um sensor no couro cabeludo, medindo os sinais de ondas cerebrais e processando-os em sinais digitais. A tecnologia torna essas medições e sinais disponíveis para jogos e aplicativos (Bos et al (2019a).

Segundo Carvalho Junior (2012), em estudos dessa natureza, pequenas amostras apresentam resultados significativos em função da ativação dos mesmos núcleos cerebrais de todos os participantes, o que poderia ser atestado em meta análise envolvendo diversos estudos ao longo do tempo, segundo constatado em Harbauch, Mayr \& amp; Burghart (2007).

A Revisão Literária nos traz informações relativas às aplicações dos sensores utilizados em EEGs em diversas áreas tais como: tecnologias assistivas, educação e treinamento, controle de máquinas, visitas virtuais entre outros. Neste trabalho abordaremos a Educação e Treinamento. Na Figura 1 são mostradas as características da Teoria Cognitiva da Aprendizagem Multimídia de Mayer.

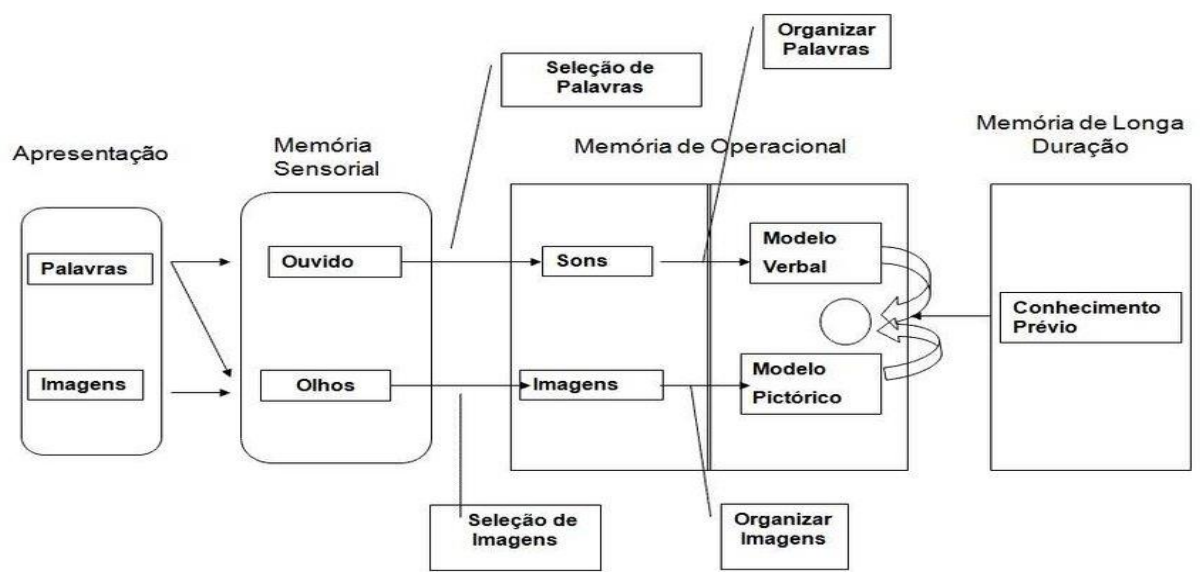

Figura 1. Elementos e interações da Teoria Multimídia de Mayer, 2008

\subsection{Hipóteses da pesquisa}

Com base na revisão de literatura, propomos hipóteses para medir a eficácia da aprendizagem em vídeos interativos por meio da análise de biosinais com técnicas de EEG. Há uma diferença significativa positiva nas ondas cerebrais dos estudantes que utilizam o método interativo e o método clássico (vídeo comum)?

\section{Material e Métodos}

Para saber o grau de contribuição que o sistema de leituras cerebrais, juntamente com o sensor de biosinais, estamos propondo investigar os tipos de atenção dos estudantes durante o estudo. Para capturar as ondas cerebrais, usamos o mindwave Neurosky que contém um chip EEG TGAM (Think Gear AM) que coleta as ondas cerebrais na forma 
de pulsos elétricos. Na Figura 2 é apresentada o fluxograma do processo da captura da leitura dos biosinais.

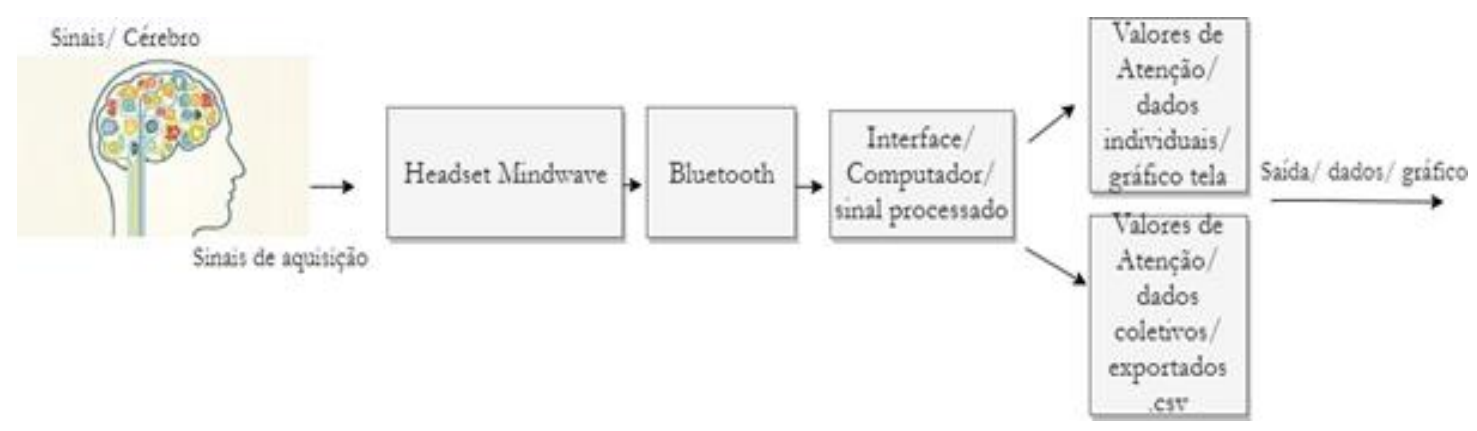

Figura 2. Fluxograma do processo de captura dos sinais.

Os pulsos elétricos são produzidos na cabeça sempre que dois neurônios se comunicam (Rebolledo-Mendez,2009). Este estudo utiliza a plataforma web H5P, para enriquecer videos de aprendizagem com diferentes métodos de comunicação e interação. O H5P oferece os seguintes métodos de interação; Perguntas simples; perguntas de múltipla escolha. Como o H5P necessita de usuários autenticados, existem o usuário aluno, professor e o pesquisador. A tela do H5P ao reproduzir um vídeo de aprendiza ge $\mathrm{m}$ mostra as barras laterais que fornece elementos de controle para invocar interações. Quando ocorre a interação o vídeo é pausado e não é possível continuar a reprodução até que o estudante reaja a interação. Nesse caso a pergunta de múltipla escolha deverá ser respondida. Na Figura 3 são mostrados os elementos com as interações propostas.

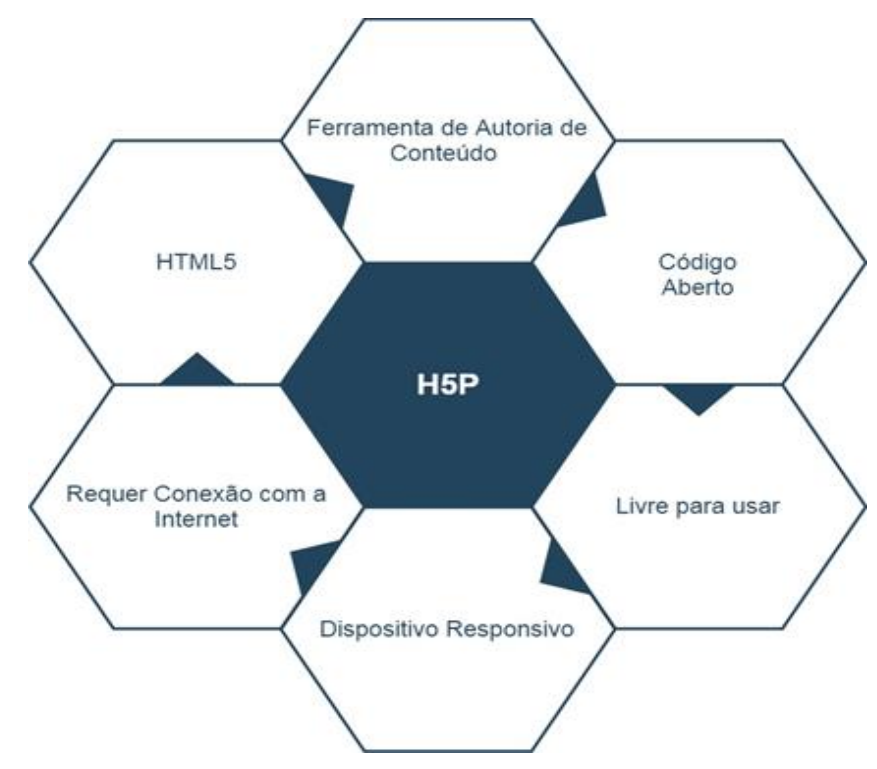

Figura 3. Elementos do H5P com as interações propostas

Durante o processo de criação, o professor pode selecionar um vídeo de várias fontes e enriquecê-los com interações, selecionando os métodos a oferecer. Por exemplo, é possível adicionar perguntas em posições predefinidas no vídeo. 
Para possibilitar a comparabilidade, o mesmo teste foi realizado para alunos que haviam sido ensinados com o material de mídia tradicional. Ambos estiveram em um nível comparável antes do estudo, com requisitos necessários para fazer uma possível comparabilidade. O teste foi construído pelos pesquisadores envolvidos na produção dos vídeos, assim as perguntas de múltipla escolha não foram elaborados por pessoa tendenciosa que possa ter influência no resultado. Foram inseridas questões referentes ao processo de ensino. Portanto, ambos tiveram a mesma posição inicial para o estudo.

\subsection{Ambiente de estudo}

A pesquisa foi realizada com grupos de estudo com ênfase em ciências biológicas. A grande maioria dos alunos tem entre 20 e 27 anos de idade. Todos os vídeos contemplavam o assunto principal em ensino de ciências. $\mathrm{O}$ experimento teve como objetivo medir a atenção do aluno durante a execução de um vídeo tradicional e um vídeo interativo, ambos com o mesmo conteúdo. A estratégia de pesquisa seguiu uma abordagem de pesquisa experimental e contou com seis sujeitos envolvidos. Os primeiros estudantes usaram a abordagem de vídeo tradicional e após foram convidados a utilizar o vídeo interativo mostrado em um desktop. Os mesmos procedimentos foram utilizados para ambos.

$\mathrm{Na}$ análise dos resultados quantitativos que foram medidos pelo sistema de leituras de sinais Neuroexperimenter, juntamente com dispositivo Mindwave Neurosky, foram encontradas diferentes intensidades nas atividades cerebrais. Com os gráficos e dados fornecidos foi possível observar as medidas de atenção, meditação e as atividades neuronais em frequências elétricas no cérebro. O resultado dessa avaliação é saber se esse tipo de estudo pode estimular a atenção dos estudantes. Na Figura 4 é apresentado a imagem do sistema de coleta de dados por estudante.

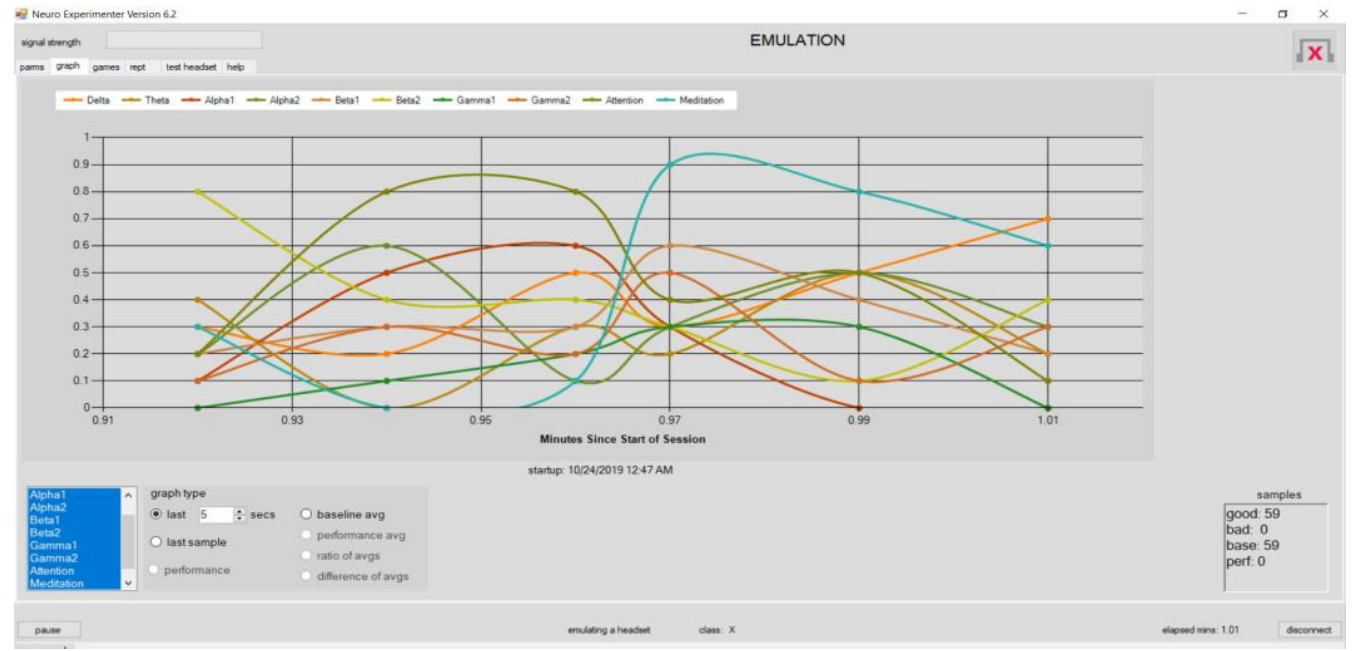

Figura 4. Sistema de coleta de dados

\section{Resultados}

Os professores de ciências estão usando essas tecnologias como um fator positivo para avaliação dos alunos. Assim, preferem utilizar diferentes tecnologias adaptadas e 
inseridas em sala de aula. É possível verificar o processo do aluno quando trabalhado o estímulo resposta.

Nos resultados é possível verificar os percentuais no gráfico, em que as cores em azul representam a medida do vídeo tradicional (método clássico). Nas cores em verde claro aparecem as percentagens da medida dos estudantes ao utilizarem o método interativo, mostrando assim o potencial positivo em relação ao método clássico. O tempo também foi observado durante o estudo, que foi de 5 minutos para cada estudante, considerando a técnica de microvídeos educacionais. A diferença entres as medidas do método interativo é observada principalmente com um grande diferença de oscilação positiva nos estudantes A5 e A6. Na Figura 5 são mostradas as médias dos dados coletados dos estudantes durante o estudo.

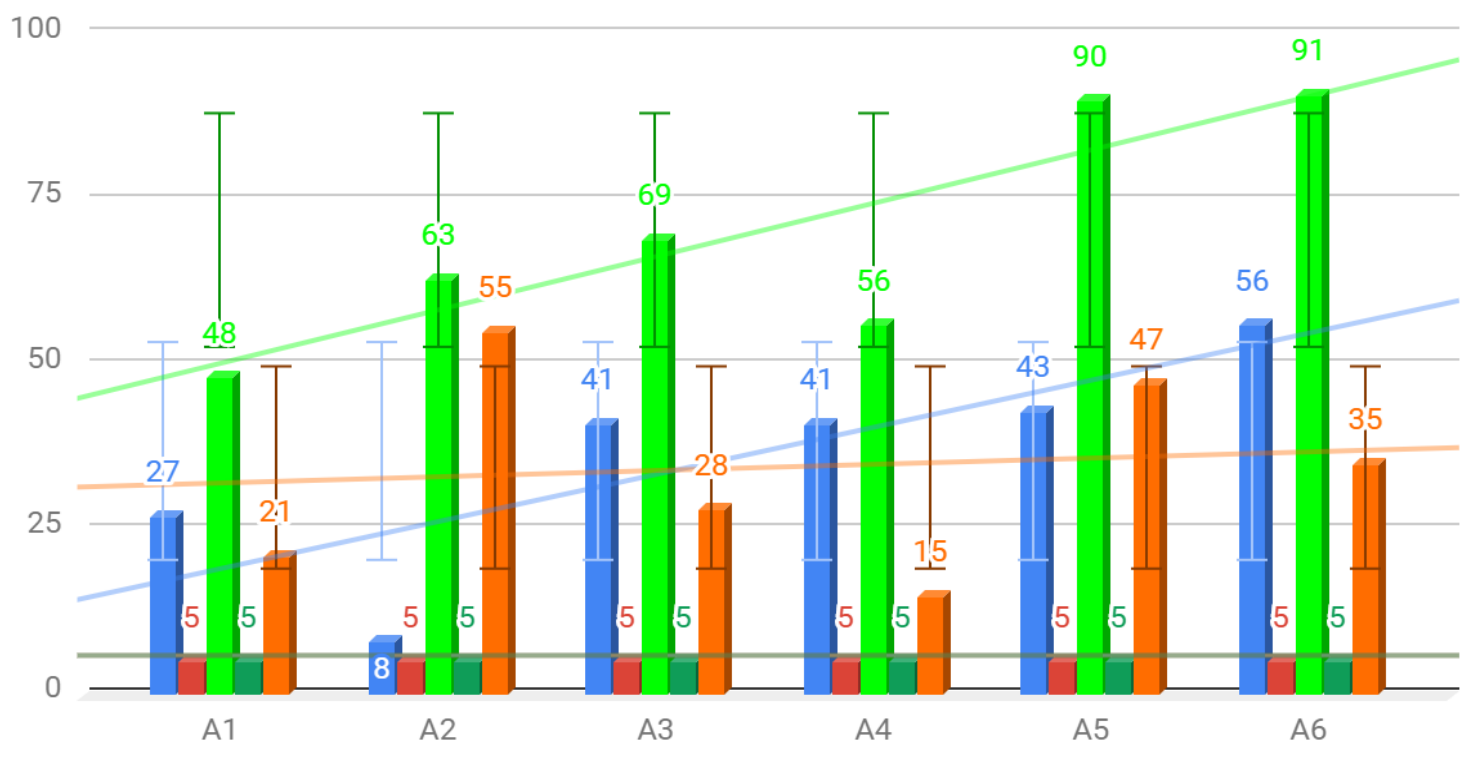

Figura 5. Dados coletados dos Estudantes

Em busca dessa resposta, exploramos os processos de atenção aprimorados pela tecnologia. Na discussão é possível evidenciar que alinhado com os resultados do estudo existe a educação centrada no aluno que pode ser melhor trabalhada.

Durante o gerenciamento dos conteúdos propostos foi possível verificar no gráfico mostrado que o vídeo interativo foi mais efetivo, quando comparado a mídia tradicional. A técnica de leitura de biosinais é mostrada nos níveis de atenção dos estudantes, assim mudanças significativas podem serem utilizadas em sala de aula adaptando estratégias de avaliação dos estudantes. É possível ainda verificar relatórios mais precisos de pontuação das ondas cerebrais e em que tempo e minuto os estudantes estavam mais atentos. Percebe-se ainda que métodos mais convencionais, que foi o uso do vídeo tradicional já 
estão ficando em desuso e não tanto eficaz nos processos de aprendizagem. Na Figura 6 são mostradas as imagens de alguns experimentos.

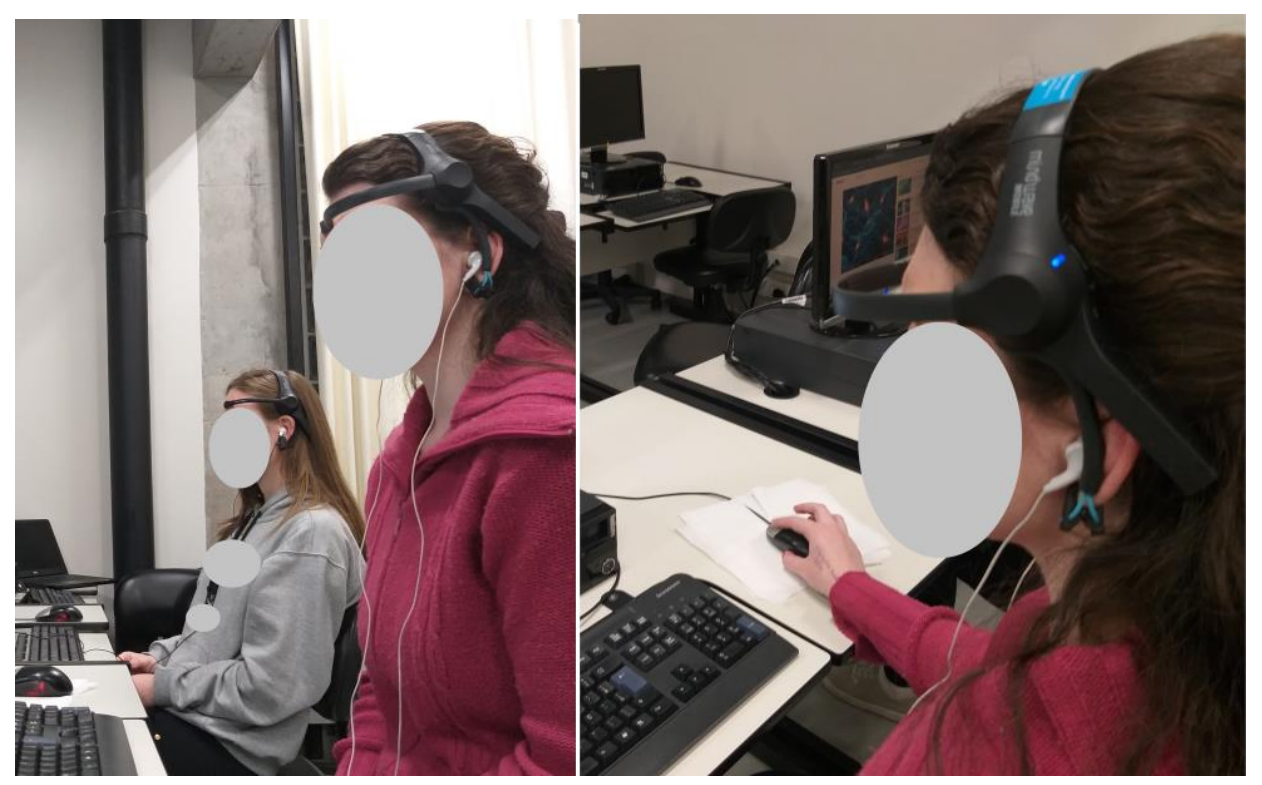

Figura 6. Experimento com os Estudantes

\section{Conclusões}

Este estudo explorou a eficácia do vídeo interativo no desempenho, satisfação e na atenção dos alunos em comparação com a mídia tradicional. Os resultados mostram que o vídeo interativo é eficaz em comparação com o vídeo comum. Além disso, o vídeo interativo teve um desempenho melhor que o vídeo comum na atenção do estudante utilizando a tecnologia de coleta de dados via sensor mindwave.

A investigação tratou da aplicação de vídeos convencionais e vídeos interativos em alunos voluntários para a pesquisa. Para isso, videos de aprendizado foram criados e enriquecidos com diferentes perguntas de múltipla escolha. Com o estudo atual, pode se mensurar a precisão das hipóteses examinadas levando em consideração as investigações da atenção do estudante. Com base nas avaliações investigadas é mostrado que perguntas de múltipla escolha durante a visualização do vídeo interativo, interferem na atenção sustentada do estudante. É mostrado ainda que perguntas que aparecem muito cedo são propensas a serem respondidas incorretamente. Portanto, identificou-se que o estudante que visualizou o vídeo convencional não obteve esse tipo de atenção. Aproximadame nte quinze segundos provou ser um tempo adequado para ocorrer a primeira pergunta interativa. Além disso, foi examinada a hipótese que afirma que as perguntas quando colocadas muito densamente uma após a outra, ocorre a maior taxa de erros.

Geralmente, resultados positivos foram alcançados durante o estudo. Estes foram examinados em uma comparação direta entre os estudantes que trabalharam com vídeos convencionais e os que trabalharam com vídeos interativos. Os estudantes com o uso da mídia interativa conseguiram obter resultados notadamente melhores.

Concluímos que o método interativo pode ser uma alternativa viável ao método clássico (vídeo comum) para estudantes. No entanto, os efeitos positivos estão situados 
na percepção do estudante com relação ao foco e na atenção situada. Como tal, este estudo pode ajudar a promover trabalhos futuros em recursos adiciona is com o uso do sensor e a utilização de vídeos por meio de melhorias na tecnologia e implantação na educação.

6. Agradecimentos: Ao Instituto Federal de Educação Ciência e Tecnologia (IFRS) e a Universidade Federal do Rio Grande do Sul (UFRGS).

\section{Referências}

BOS, A. S., HERPICH, F., KUHN, I., GUARESE, R. L. M., TAROUCO, L. M. R., ZARO, M. A.,WIVES, L. (2019). Educational Technology and Its Contributions in Students' Focus and Attention Regarding Augmented Reality Environments and the Use of Sensors. Journal of Educational Computing Research. vol.57, n.7 https:/doi.org/10.1177/0735633119854033

BOS et al., Student's attention: The use of Brain Waves Sensors in Interactive Videos. International Journal of Advanced Engineering Research and Science, v. 6, n. 4, 2019a.

BOS, A.; PRESTES, L.; PIZZATO M.; ZARO M. (2019b). Vídeos Educativos: Investigação da Atenção e o Mapeamento do Aprendizado Ativo. In: Abordagens teóricas e reflexões sobre a educação presencial a distância e corporativa. Maringá: Uniedusul Editora.

BOS, Andreia; ZARO, Milton Antônio; PIZZATO, Michelle. Investigação da Atenção do estudante com técnicas de EEG: o uso da Realidade Virtual no Ensino. In: Anais dos Workshops do Congresso Brasileiro de Informática na Educação. 2019c. p. 1397.

BOS, Andreia; PIZZATO, Michelle; ZARO, Milton; Investigação da atenção do estudante: o uso da realidade virtual no ensino de computação. Tear: Revista de Educação, Ciência e Tecnologia, vol 8, n. 2, Dez, 2019d. Disponível em: https:/periodicos.ifrs. edu.br/index.php/tear/index

BRUNER, J. (1986). Actual minds, possible worlds. Cambridge, Massachusetts: Harvard University Press

CHEN, C. M., \& LIN, Y. J. (2014). Effects of different text display types on reading comprehension, sustained attention and cognitive load in mobile reading contexts. Interactive Learning Environments http://dx.doi.org/10.1080/10494820.2014.891526.

EFFECTIVE LEARNER. (2019). Página oficial. Disponível em: <https://store.neurosky.com/ products/effective-learner>. Acesso em setembro, 2019. GUO PJ, KIM J, AND ROBIN R (2014). How video production affects student engagement: An empirical study of MOOC videos. ACM Conference on Learning at Scale (L@S 2014); found at http//groups.csail.mit.edu/uid/other-pubs/las2014-pguoengagement.pdf.

HERPICH, F.; BOS, A.; KUHN, I.; GUARESE, R.; TAROUCO, L.; WIVES, L.; ZARO, M. Atividade cerebral no uso de recursos educacionais em realidade aumentada: uma 
análise da atenção do aprendiz In: XXIX Simpósio Brasileiro de Informática na Educação (Brazilian Symposium on Computers in Education), 2018, Fortaleza. org.crossref.xschema._1.Title@772293c4. ,2018.p.1858.

IZQUIERDO I., C.R.G. FURINI, J.C. Myskiw Fear memory Physiological Reviews, 96 (2) (2016), pp. 695-750, 10.1152/physrev.00018.2015

KREINER DS (1997). Guided notes and interactive methods for teaching with videotapes. Teaching of Psychology 24, 183-185.

LENT, R.(2015) Neuroplasticidade. In: LENT, Roberto (Org.). Neurociência da mente e do comportamento. Rio de Janeiro: Guanabara Koogan. p. 241-252.

MA \& WEI (2016), A comparative study of children's concentration performance on picture books: Age, gender, and media forms Interactive Learning Environments, 24 (8)(2016), pp. 1922-1937

MACKENZIE, L., \& BALLARD, K. (2015). Can using individual online interactive activities enhance exam results? Journal of Online Learning and Teaching, 11(2), 262266. , p. 266)

MAYER R. (2008). Applying the science of learning: Evidence-based principles for the design of multimedia instruction. Cognition and Instruction 19, 177-213.

MINDWAVE MOBILE. (2019). Página Oficial. Disponível em: $<$ http $/ /$ store.neurosky.com/ products/mindwave-mobile $>$. Acesso em setembro, 2019.

MORA, F. (2013). Neuroeducación, Solo se puede aprender aquello que se ama. Madrid: Alianza Editorial.

PAVLOV, IVAN. (1927). Conditioned reflexes: An investigation of the physiological activity of the cerebral cortex.

RAMOS, Guilherme; DA SILVA, Patrícia Grasel. Elementos pré-textuais e comitês dos Workshops do CBIE 2019. In: Anais dos Workshops do Congresso Brasileiro de Informática na Educação. 2019.

REBOLLEDO-MENDEZ, G., DUNWELL, I., MARTÍNEZ-MIRON, E., VARGASCERD AN, M., DE FREITAS, S., LIAROKAPIS, F., et al., (2009). Assessing NeuroSky's usability to detect attention levels in an assessment exercise. HCI, new Trends (pp. 149 e 158)

TOKUHAMA-ESPINOSA, T. N. (2008). The scientifically substantiated art of teaching: A study in the development of standards in the new academic field of neuroeducation (mind, brain, and education science). Capella University, MN, USA.

XIAO, Tianjun et al., The application of two-level attention models in deep convolutional neural network for fine-grained image classification. In: Proceedings of the IEEE Conference on Computer Vision and Pattem Recognition. 2015. p. 842-850. 